Eva Dombi, MD

Simone L. Ardern-

Holmes, MD

Dusica Babovic-

Vuksanovic, MD

Fred G. Barker, MD

Steve Connor, MD

D. Gareth Evans, MD

Michael J. Fisher, MD

Stephane Goutagny, MD, $\mathrm{PhD}$

Gordon J. Harris, PhD

Diego Jaramillo, MD

Matthias A. Karajannis, MD

Bruce R. Korf, MD, PhD

Victor Mautner, MD

Scott R. Plotkin, MD, $\mathrm{PhD}$

Tina Y. Poussaint, MD

Kent Robertson, MD,

$\mathrm{PhD}$

Chie-Schin Shih, MD

Brigitte C. Widemann, MD

For the REiNS

International

Collaboration

Correspondence to

Dr. Dombi:

dombie@mail.nih.gov

Supplemental data at www.neurology.org

\title{
Recommendations for imaging tumor response in neurofibromatosis clinical trials
}

\section{ABSTRACT}

Objective: Neurofibromatosis (NF)-related benign tumors such as plexiform neurofibromas (PN) and vestibular schwannomas (VS) can cause substantial morbidity. Clinical trials directed at these tumors have become available. Due to differences in disease manifestations and the natural history of NFrelated tumors, response criteria used for solid cancers (1-dimensional/RECIST [Response Evaluation Criteria in Solid Tumors] and bidimensional/World Health Organization) have limited applicability. No standardized response criteria for benign NF tumors exist. The goal of the Tumor Measurement Working Group of the REiNS (Response Evaluation in Neurofibromatosis and Schwannomatosis) committee is to propose consensus guidelines for the evaluation of imaging response in clinical trials for NF tumors.

Methods: Currently used imaging endpoints, designs of NF clinical trials, and knowledge of the natural history of NF-related tumors, in particular PN and VS, were reviewed. Consensus recommendations for response evaluation for future studies were developed based on this review and the expertise of group members.

Results: MRI with volumetric analysis is recommended to sensitively and reproducibly evaluate changes in tumor size in clinical trials. Volumetric analysis requires adherence to specific imaging recommendations. A $20 \%$ volume change was chosen to indicate a decrease or increase in tumor size. Use of these criteria in future trials will enable meaningful comparison of results across studies.

Conclusions: The proposed imaging response evaluation guidelines, along with validated clinical outcome measures, will maximize the ability to identify potentially active agents for patients with NF and benign tumors. Neurology ${ }^{\circledR}$ 2013;81 (Suppl 1):S33-S40

\section{GLOSSARY}

$\mathbf{C R}=$ complete response; $\mathbf{N F}=$ neurofibromatosis; $\mathbf{P D}=$ progressive disease; $\mathbf{P N}=$ plexiform neurofibroma; $\mathbf{P R}=$ partial response; RECIST = Response Evaluation Critera in Solid Tumors; REiNS = Response Evaluation in Neurofibromatosis and Schwannomatosis; SD = stable disease; $\mathbf{S T I R}=$ short TI inversion recovery; TTP = time to progression; $\mathbf{V S}=$ vestibular schwannoma; WHO = World Health Organization.

Standard criteria for response evaluation in clinical trials for solid tumors are well established (World Health Organization [WHO] criteria, Response Evaluation Criteria in Solid Tumors [RECIST]). ${ }^{1,2}$ Linear measurements are performed for their ease of use and are suitable for most malignant lesions that rapidly change in size. Disease-specific recommendations have been developed for some diseases in which linear measurements are not practical or meaningful, such as the Response Assessment in Neuro-Oncology (RANO) criteria for brain tumors. ${ }^{3,4}$ In addition, an international effort is under way to develop measurement guidelines for pediatric brain tumors, which will be applicable to neurofibromatosis type 1 (NF1)-related gliomas.

\footnotetext{
From the Pediatric Oncology Branch (E.D., B.C.W.), National Cancer Institute, Bethesda, MD; Department of Neurology (S.L.A.-H.), The Children's Hospital at Westmead, Sydney, Australia; Department of Medical Genetics (D. B.-V.), Mayo Clinic, Rochester, MN; Neurosurgical Service (F.G.B.), Department of Radiology (G.J.H.), and Department of Neurology and Cancer Center (S.R.P.), Massachusetts General Hospital, Boston, MA; Department of Neuroradiology (S.C.), King's College Hospital, London, UK; Department of Genetic Medicine (D.G.E.), MAHSC, St Mary's Hospital, Manchester, UK; Division of Oncology (M.J.F.) and Department of Radiology (D.J.), The Children's Hospital of Philadelphia; Department of Pediatrics (M.J.F.), The Perelman School of Medicine at the University of Pennsylvania, Philadelphia, PA; Department of Neurosurgery (S.G.), Hôpital Beaujon, Clichy, France; Division of Pediatric Hematology/Oncology and NYU Cancer Institute (M.A.K.), NYU Langone Medical Center, New York, NY; Department of Genetics (B.R.K.), University of Alabama at Birmingham, Birmingham, AL; Department of Neurology (V.M.), University Medical Center Hamburg-Eppendorf, Hamburg, Germany; Department of Radiology (T.Y.P.), Boston Children's Hospital, Boston, MA; and Department of Pediatrics (K.R., C.-S.S.), Riley Hospital for Children, Indianapolis, IN. REiNS International Collaboration members are listed on the Neurology ${ }^{\circledR}$ Web site at www.neurology.org. Go to Neurology.org for full disclosures. Funding information and disclosures deemed relevant by the authors, if any, are provided at the end of the article.
} 
The neurofibromatoses (NF), consisting of NF1, neurofibromatosis type 2 (NF2), and schwannomatosis, are genetic tumor predisposition syndromes characterized by the development of predominantly benign nerve sheath tumors. Plexiform neurofibromas (PN) in NF1, vestibular schwannomas (VS) and meningiomas in NF2, and schwannomas in NF2 and schwannomatosis are the most frequent histologically benign tumors for which clinical trials with targeted agents have been initiated. Due to the limited applicability of response criteria for solid tumors and the lack of standardized response criteria for $\mathrm{NF}$ tumors, previous trials have used a variety of primary and secondary endpoints, which limits comparison of results between trials.

The Response Evaluation in Neurofibromatosis and Schwannomatosis (REiNS) Tumor Measurement Group was formed to develop standardized consensus recommendations for imaging response evaluation in clinical trials for benign NF tumors. This includes guidelines for image acquisition, target lesion selection, image interpretation by volumetric analysis, and trial design. We hope that the application of these criteria in future clinical trials will

\section{Figure 1 Comparison of measurement sensitivity to detect change in tumor size}

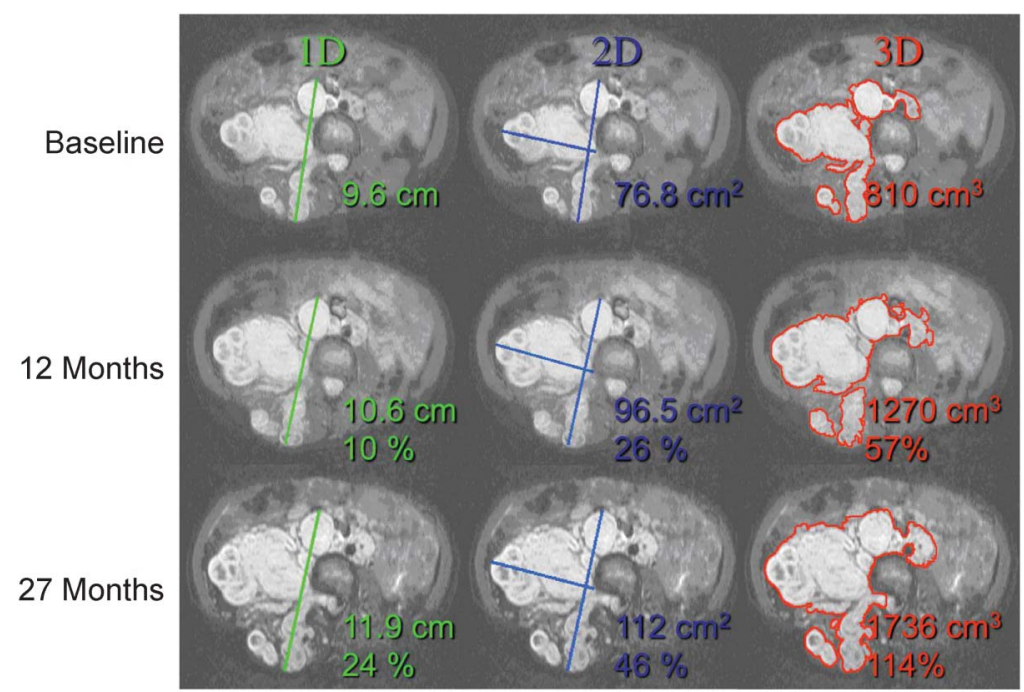

Axial short TI inversion recovery MRI images show a large paraspinal, abdominal, and pelvic plexiform neurofibroma at 3 time points (top to bottom: baseline, 12 months, 27 months) measured by 3 methods (left to right: 1D/RECIST, 2D/WHO, 3D/volumetric criteria). Progressive disease was detected after 27 months by RECIST criteria, after 12 months by WHO criteria, and after 6 months by the proposed volumetric criteria ( $20 \%$ volume increase, not shown). At the time of $2 \mathrm{D}$ progression, the volume increase was $57 \%$, and by the time of 1D progression, the volume had more than doubled. RECIST = Response Evaluation Criteria in Solid Tumors; WHO = World Health Organization. promote effective evaluation of the activity of novel agents and facilitate the comparison of results across trials.

METHODS The Tumor Measurement Group is composed of members from different disciplines, including neurology, oncology, radiology, and genetics. The group reviewed currently used imaging endpoints, designs of NF clinical trials, and the knowledge of the natural history of NF-related tumors, in particular PN and VS. Consensus guidelines for future studies were developed based on this review and the expertise of group members. NF-related tumors are rare and there was an emphasis on proposing criteria that could be applied across multiple participating sites. When feasible, the RECIST guidelines were used as a template.

RESULTS Current response evaluation of benign NF tumors in clinical trials. PN involve multiple nerve fascicles and branches ${ }^{5}$ and can cause substantial morbidity, including pain, disfigurement, motor dysfunction, airway compromise, and vision loss. ${ }^{6,7}$ In early clinical trials PN size was measured by RECIST or WHO criteria. ${ }^{8,9}$ Due to the complex shape, large size, and slow growth of $\mathrm{PN}$, linear measurements can be highly variable and long time periods are required to detect measurable change (figure 1). Therefore, more sensitive methods, such as volume segmentation, were developed for the analysis of PN and schwannomas. ${ }^{10-15}$ These methods documented the feasibility of reproducibly measuring tumor volumes in natural history studies and treatment trials for PN and VS..$^{13,14,16-19}$ Using the MEDx-based lesion detection method developed for PN, interobserver differences were less than $10 \%$ and intraobserver variation was $\leq 5 \% .{ }^{10}$ For VS measured using the Vitrea2 workstation, the coefficient of variation ranged from $0.6 \%$ to $6.8 \% .^{11}$

The first PN clinical trial using volumetric MRI analysis with time to progression (TTP) as the primary trial endpoint included the comparison of 1-dimensional (RECIST), 2-dimensional (WHO), and volumetric MRI analysis of PN. ${ }^{20}$ Volumetric analysis detected tumor progression ( $\mathrm{PN}$ volume increase $\geq 20 \%$ ) much earlier than linear measurements. The median TTP with volumetric analysis was 14.3 months, compared to 52.2 months using WHO criteria; the median TTP could not be determined by RECIST criteria. ${ }^{20}$ Thus, volumetric analysis significantly shortened both drug exposure for study subjects and the duration of the trial. In addition, this study provided valuable natural history data on PN growth. Volume increase over time appeared to be linear for all patients, with younger patients experiencing more rapid growth. The inverse relationship between age and PN growth rate has been confirmed in other studies. ${ }^{21-23}$ Spontaneous PN shrinkage was not observed in the placebo arm of the study and has only infrequently been observed in other studies. ${ }^{22}$

NF2 is characterized by the development of bilateral VS, other intracranial and spinal schwannomas, 
meningiomas, and ependymomas. ${ }^{24-26}$ In large NF2associated VS natural history studies, the average growth in greatest diameter per year was reported to be between 1.3 and $1.8 \mathrm{~mm}$, corresponding to a $10 \%-14 \%$ increase from the baseline averages of 12.4 and $13.0 \mathrm{~mm} \cdot{ }^{27,28}$ Another study evaluated the feasibility of volumetric assessment of VS and demonstrated increased sensitivity in detecting disease progression and reduced intrarater measurement variability compared to linear measurements. ${ }^{11}$ Finally, one study described the pattern of volume increase of VS and meningiomas as saltatory for the majority of tumors and linear or exponential for others. ${ }^{29}$ Volumetric analysis of MRIs has been recommended to determine changes in tumor size $e^{30}$ and has been incorporated into clinical trials targeting NF2related VS. ${ }^{17,18,31}$

Guidelines for response evaluation of benign NF tumors in clinical trials. Image acquisition and analysis. The working group recommends MRI and volumetric analysis to measure benign NF-related tumors and to assess response in clinical trials. Excellent tissue definition can be achieved with appropriate MRI techniques. Clinical trials require sequential imaging with fairly short intervals. Subjects with benign tumors remain in studies for much longer time periods than those with malignant solid tumors and have a longer life expectancy. ${ }^{6}$ It is therefore preferable to avoid CT imaging, as serial imaging results in considerable radiation exposure and the technique has not been validated for volumetric analysis of NF tumors.

Volumetric MRI analysis accounts for every part of the tumor and thus reflects the actual size of the lesion more closely than linear measurements. It is less sensitive to differences in body position between scans and can reproducibly detect small changes over time (figure 2 ). Since every tumor-containing slice is included in the analysis, volumetric analysis requires excellent image quality. In order to achieve consistent high-quality
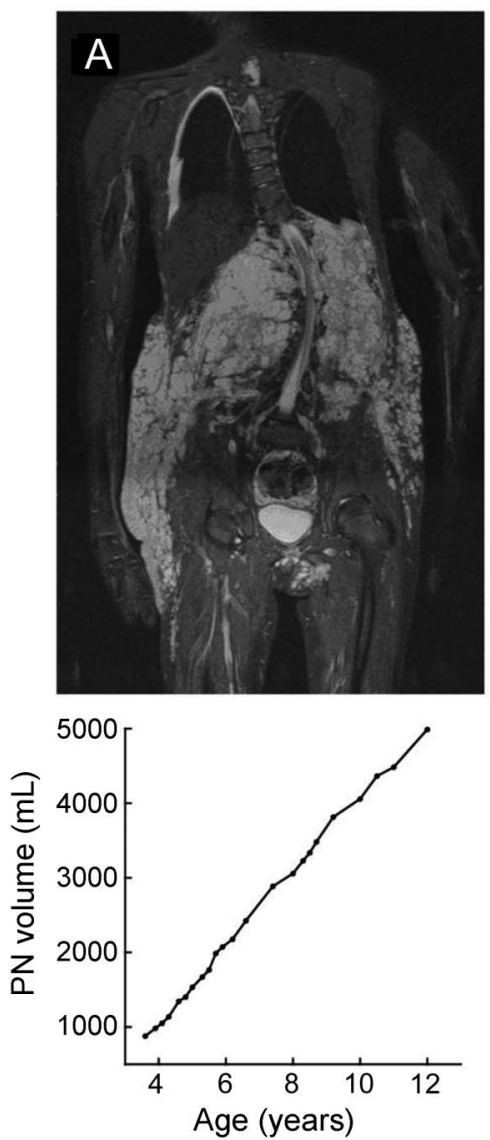
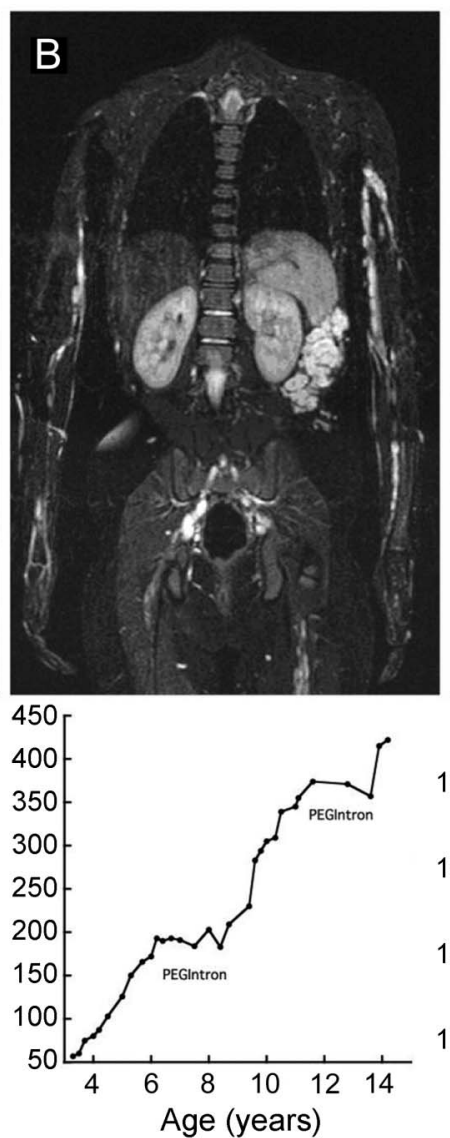
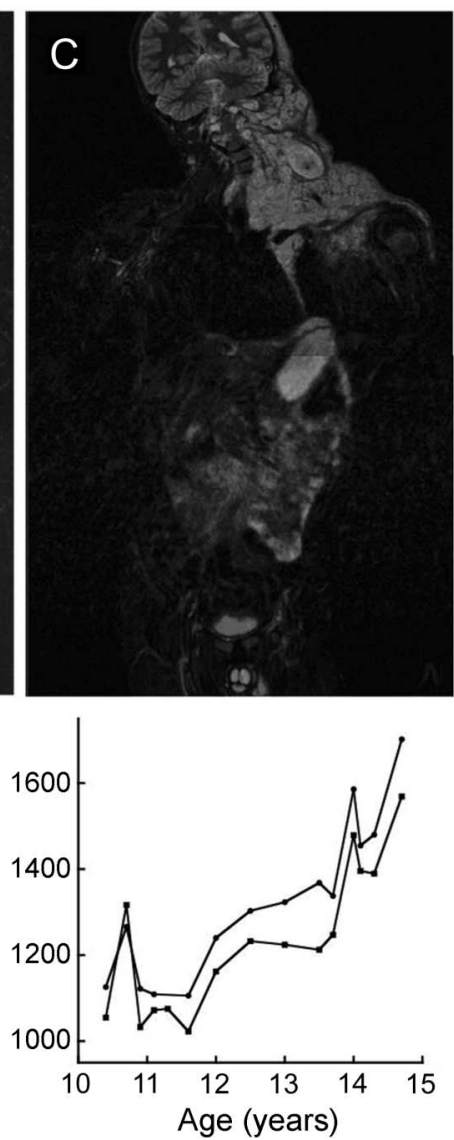

Small incremental changes are demonstrated in a complex large abdominal and flank plexiform neurofibroma (PN) with consistent upward trend through several investigational treatments (A). Extended disease stabilization of a back PN in a patient receiving peginterferon alfa- $2 b(B)$. $P N$ growth accelerated upon discontinuation of treatment and slowed again when the patient restarted peginterferon alfa-2b. While in most cases $\mathrm{PN}$ growth is linear over extended periods, some measurement variation is not uncommon, as demonstrated by the example of a large neck and chest PN (C). The first observer performed the volumetric analysis prospectively and a second observer repeated the analysis at the end of the observation period while blinded to the chronological order of the scans. 
image acquisition and to minimize the variability of volumetric readings, a detailed section on image acquisition should be included in study protocols.

The MRI acquisition protocol should be optimized for tumor type and tumor location. Images intended for volumetric analysis need to be performed without gaps between slices. The target tumor should be positioned close to the center of the imaging field and the outer edge of the tumor should be within the field of view. Peripheral nerve sheath tumors can be well visualized without the use of contrast agents on short TI inversion recovery (STIR) sequences because they have high signal intensity relative to normal tissues. Small lesions, such as some orbital, facial, or paraspinal tumors, require imaging with thin slices using small voxel sizes. Obtaining complete coverage of extensive lesions can be challenging. MRI scanners capable of reconstructing imaging series from several body segments into whole-body images are now available and whole-body scanning is increasingly affordable. Using multiple phased-array coil technology, whole-body STIR imaging can be accomplished in less than an hour. Tumor volume can also be calculated from independent series. For best alignment of separate series, 2 to 5 slices of complete overlap between the series is advised. Image orientation should remain the same throughout the study. Even slight changes in orientation can result in partial overlap between series. STIR sequences can be performed on $1.5 \mathrm{~T}$ or $3 \mathrm{~T}$ MRI scanners, although magnetic field inhomogeneity resulting in signal intensity variation is more common using 3T magnets when imaging with large fields of view.

The challenge in imaging VS is their relatively small size. High-resolution postcontrast T1 completely covering the target area is the MRI sequence best suited for volumetric measurement. The slice thickness should be no more than $1 \mathrm{~mm}$. Fat suppression is required in postsurgical VS cases to differentiate tumor from fat packing.

Table e-1 on the Neurology ${ }^{\circledR}$ Web site at www. neurology.org provides examples of recommended imaging parameters for PN and VS. The parameters may vary depending on the type of scanner, tumor type, and location, but consistent use at each response evaluation and across multiple sites is required for optimal analysis. In multicenter studies, it is important for quality assurance to document each site's ability to comply with the imaging protocol prior to starting enrollment. One way to achieve identical image acquisition across participating sites is to distribute an optimized sample Digital Imaging and Communications in Medicine dataset and duplicate the imaging parameters from that dataset at each site. We plan to provide access to sample images on the REiNS Web site www.reinscollaboration.org.
Target lesions: Identification, considerations and challenges. Patients with NF often present with more than one symptomatic tumor. Ideally the entire tumor burden should be imaged at study entry. In most cases, the most clinically significant lesion can be identified and used as the target lesion to evaluate treatment response, and other disease sites can be monitored as nontarget lesions. The target lesion should meet minimum size criteria, be seen on at least 3 imaging slices, and have reasonably well-defined contours in all dimensions. Recommended minimum tumor sizes for measurable disease are $3 \mathrm{~cm}^{3}$ for $\mathrm{PN}$ and $1 \mathrm{~cm}^{3}$ for VS. Most lesions $\geq 3.0 \mathrm{~cm}$ in longest diameter will have a volume greater than $3 \mathrm{~cm}^{3}$, and lesions $\geq 1.5 \mathrm{~cm}$ a volume over $1 \mathrm{~cm} .{ }^{3}$ Central review can help in assessing the measurability of questionable lesions. The target lesion should be distinguishable from the surrounding tissues. Some tumors, while clinically very relevant, may not have good tissue definition on MRI (figure 3, A and B). In case of disease progression, adjacent tumors can become confluent and no longer independently measurable (for example, collision tumors in NF2 VS). In addition, adjacent lymph nodes can be difficult to separate from $\mathrm{PN}$ and are prone to size fluctuations, enough to give the impression of significant volume change if the target lesion is small.

Lesions previously treated with locoregional therapy such as radiation should not be selected as target lesions unless growth has been documented since the intervention. Similarly, following partial tumor resection, postsurgical changes and edema may interfere with meaningful response evaluation (figure 3, C and D).

Motion can blur the edge of lesions; regions especially prone to motion include the parapharyngeal space, diaphragm, mesenteric structures, and extremities. Motion artifact is most relevant for small tumors with high surface to volume ratios; at tumor sizes above $100 \mathrm{~cm}^{3}$ the effect of motion is usually negligible.

Metal instrumentation, such as spinal rods, dental braces, or cochlear/brainstem implants, causes characteristic distortion on MRI that is dependent on the magnetic field strength and positioning within the scanner, and can therefore vary between scans (figure $3, \mathrm{E}$ and $\mathrm{F}$ ). If part of the target tumor is obscured or distorted by metallic artifact, the tumor may still be measurable as long as that part of the lesion can consistently be excluded from the analysis. The potential need for future surgery or metal implants should be considered prior to enrollment in a trial.

For very large tumors, partial volume measurement between reproducibly visualized anatomical landmarks can be considered, but should aim to include the bulk of the lesion.

Some PN have nodular components that appear encapsulated and separated from the surrounding tumor in all directions (figure 4). These nodules can 
Figure 3 Considerations for the selection of target lesions for volumetric assessment
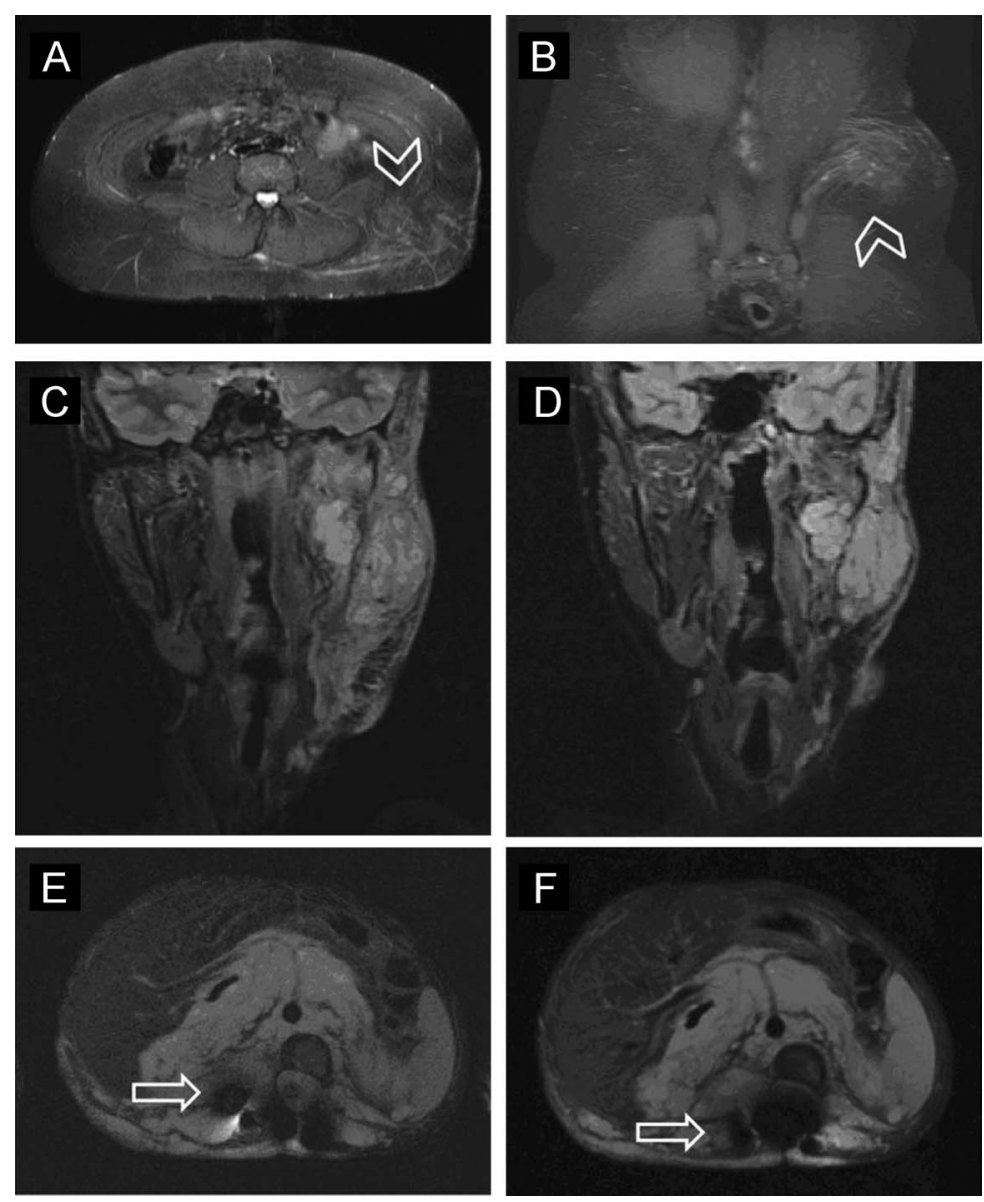

Volumetric analysis is only feasible on lesions with well-defined borders. The superficial flank plexiform neurofibroma (PN) shown on axial (A) and coronal (B) short TI inversion recovery MRI lacks tissue contrast (arrowheads) and it is not suitable for volume measurement. Edema within a tumor or surrounding tissue affects the lesion volume $(C, D)$. Image $C$ shows a facial PN 2 months after debulking surgery. The tumor volume gradually decreased over the next 10 months until the complete resolution of postsurgical changes (D). Metal in the imaging field results in image distortion on MRI (arrows), and the position of metal artifact may vary between scans $(E, F)$.

be independently measured and may serve as target lesions. The growth rate of nodular lesions may exceed the growth rate of the surrounding tumor. ${ }^{32}$ If the nodular component is selected as the target lesion, changes in that nodule as opposed to the entire PN should be used to determine response. Highly disproportionate growth within parts of a tumor or rapid growth exceeding that expected for PN should raise concern for malignant transformation and prompt further investigation.

Image interpretation by volumetric analysis. Several methods are available for volumetric evaluation of medical images. Simple systems use a graphic tool to manually outline the target area. More sophisticated segmentation methods use complex algorithms to define a region of interest that displays specific imaging characteristics; some of these methods were specifically developed for NF-related tumors. ${ }^{10-13,15}$
Volumetric results can differ based on the method of analysis; therefore, the same method should be used throughout a study and across all participating institutions, ideally with central review. The analysis method should be validated to determine variation within the same dataset as a reflection of precision, and variation in trends over time as an indicator of accuracy in segmenting the same structures at different time points.

Response criteria. Response categories are established to differentiate tumors that are shrinking, stable, or growing. These categories are arbitrary and were designed to minimize interrater variability. Significant changes in tumor size, as defined by RECIST or WHO criteria, can be easily recognized. Targeted agents in NF may not result in substantial tumor shrinkage, but rather lead to disease stabilization or minor shrinkage. The goal of volumetric MRI analysis for response evaluation in NF is to reproducibly detect small changes that would not be otherwise discernible. Based on the close intraobserver and interobserver agreement of repeated volumetric MRI analyses, ${ }^{10,11}$ the group agreed on a $20 \%$ volume change to indicate a decrease or increase in tumor size. Therefore, the recommended volumetric response categories for benign NF tumors are defined as follows:

- Complete response (CR): Disappearance of the target lesion.

- Partial response (PR): Decrease in the volume of the target lesion by $20 \%$ or more compared to the baseline. The PR is considered unconfirmed at the first detection, confirmed when observed again within 3-6 months, and sustained when the response is maintained for 6 months or longer.

- Progressive disease (PD): Increase in the volume of the target lesion by $20 \%$ or more compared to baseline or the time of best response after documenting a PR. The appearance of new lesions or unequivocal progression of existing nontarget lesions is also considered PD.

- Stable disease (SD): Insufficient volume change to qualify for either PR or PD.

Table 1 illustrates equivalent size changes in spherical lesions using different measurement methods.

Considerations for trial design and selection of endpoints using volumetric response evaluation. Many of the molecularly targeted agents evaluated for the treatment of NF-related tumors are thought to be more likely to extend the TTP than to result in tumor shrinkage. Progression-free survival as a study endpoint requires a control population. There is no established historical control for all NF populations. Data from one placebo-controlled pediatric trial for PN are available and are currently used as a historical control for 

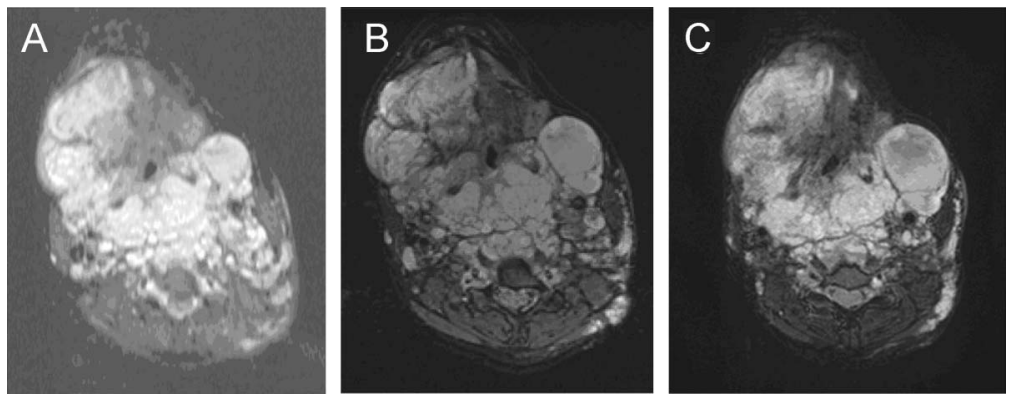

The diffuse face and neck plexiform neurofibroma shown on axial short TI inversion recovery MRI has a well-circumscribed nodular component on the left side. The complete volume of the lesion increased from $1,870 \mathrm{~mL}$ at the first time point (A) to $2,015 \mathrm{~mL}$ ( $8 \%$ increase) at the second time point (B) to $2,283 \mathrm{~mL}$ ( $22 \%$ increase) on the final evaluation (C). Concurrently, the volume of the nodular component increased from $28.2 \mathrm{~mL}$ to $34.3 \mathrm{~mL}$ (22\% increase) and then to $63.0 \mathrm{~mL}$ ( $84 \%$ increase). Determination of disease progression depends on whether the entire lesion or the nodular component is selected as the target lesion at trial entry.

several other studies. ${ }^{33,34}$ This control population is only valid for trials with identical eligibility criteria and study design, as TTP is influenced by age, tumor growth rate, tumor size, and restaging intervals. ${ }^{20}$

Due to the slowly progressive nature of most NF-related tumors, prolonged stable disease without any intervention is common. Therefore, disease stability can only be considered a response in subjects with documented imaging progression of a tumor for which linear growth is expected. Ideally, progression at the time of enrollment should be documented using the same method of analysis that will be used in the clinical trial.

The need for an adequate control population and the requirement of progressive disease at the time of enrollment in a trial with TTP as the primary endpoint may affect the rate of accrual, as progression may be documented in only a subset of candidates and competing trials without a control group may be selected by investigators or potential research participants.

The objective response rate is defined as the proportion of subjects experiencing a $\mathrm{PR}$ or $\mathrm{CR}$ in a

\begin{tabular}{|c|c|c|}
\hline Table 1 & \multicolumn{2}{|c|}{$\begin{array}{l}\text { Equivalent changes in the size of } \\
\text { spherical lesions by 1-dimensional, } \\
\text { 2-dimensional, or 3-dimensional } \\
\text { measurement }\end{array}$} \\
\hline RECIST 1D (2r & WHO $2 \mathrm{D}\left(\pi \mathrm{r}^{2}\right)$ & Volume 3D $\left(4 / 3 \pi r^{3}\right)$ \\
\hline$-30 \% \mathrm{PR}^{\mathrm{a}}$ & $-50 \% \mathrm{PR}^{\mathrm{a}}$ & $-66 \%$ \\
\hline$-7 \%$ & $-14 \%$ & $-20 \% P^{a}$ \\
\hline $6 \%$ & $13 \%$ & $20 \% \mathrm{PD}^{\mathrm{a}}$ \\
\hline $12 \%$ & $25 \% \mathrm{PD}^{\mathrm{a}}$ & $40 \%$ \\
\hline $20 \% P^{a}$ & $44 \%$ & $73 \%$ \\
\hline
\end{tabular}

Abbreviations: RECIST $=$ Response Evaluation Criteria in Solid Tumors; WHO = World Health Organization.

${ }^{a}$ Change required for partial response (PR) or progressive disease (PD) as defined by RECIST, WHO, or volumetric criteria. study. A correlation between response on imaging and overall survival or clinical improvement has not been established for most benign NF tumors. If feasible, in addition to imaging response, evaluation for clinical benefit should be incorporated into clinical trials as endpoints, including patient-reported and functional outcomes. Since spontaneous regression of NF tumors is rare, imaging response can be evaluated without placebo control and in patients without a prior history of progression.

Clinical studies for NF take longer than typical cancer trials. Even with the use of sensitive detection methods, tumor progression and response may occur slowly, over years rather than months. Compared with cancer trials, longer restaging intervals are sufficient, such as every 3-4 months for the first year on trial and subsequently every 6 months. Allowing subjects with minimal tumor shrinkage at 6 months (10-15\% decrease) to remain on treatment for up to 12 months can maximize the identification of potentially active agents while minimizing exposure to inactive agents.

The natural history of NF-related tumors should also be considered in the design of trials. Several studies have described more rapid growth rates of $\mathrm{PN}$ in younger children compared to older children or adults. ${ }^{21-23}$ Therefore, stratification based on age or progression status could be considered for clinical trials evaluating TTP as the primary endpoint.

FUTURE PLANS The consensus guidelines provided here are based on current knowledge and experience. With new information, revisions may be implemented. At this time, our understanding of the natural history of NF1, NF2, and schwannomatosis is incomplete. Prior studies describing the growth pattern of NF-related tumors are limited to relatively short observation periods of $1-4$ years. Long-term prospective natural history studies are needed in order to better characterize tumor growth in all age groups and tumor types. Additional efforts are under way to study the correlation of changes in tumor size with changes in patient-reported and functional outcomes. Where a correlation between increasing tumor size and the development of morbidity can be established, it can be assumed that agents resulting in prolonged disease stabilization may result in patient benefit by preventing the development of new morbidity. Volumetric assessment methods currently in use for NF may differ in their sensitivity to detect change over time. As part of the REiNS Collaboration, the Tumor Measurement Working Group is planning pilot studies to evaluate the level of agreement between methods. Finally, volumetric MRI analysis is time and resource intensive and requires central review for the most consistent results. The development of methods that can be performed more easily and be incorporated into routine clinical practice should be pursued. 


\section{AUTHOR CONTRIBUTIONS}

E Dombi: drafting the manuscript, study concept, interpretation of data. SL Ardern-Holmes: revising the manuscript for content, study concept, interpretation of data. D Babovic-Vuksanovic: revising the manuscript for content, study concept, interpretation of data. FG Barker: revising the manuscript for content, study concept, interpretation of data. S Connor: revising the manuscript for content, study concept, interpretation of data. DG Evans: revising the manuscript for content, study concept, interpretation of data. MJ Fisher: revising the manuscript for content, study concept, interpretation of data. S Goutagny: revising the manuscript for content, study concept, interpretation of data. GJ Harris: revising the manuscript for content, study concept, interpretation of data. D Jaramillo: revising the manuscript for content, study concept, interpretation of data. MA Karajannis: revising the manuscript for content, study concept, interpretation of data. BR Korf: revising the manuscript for content, study concept, interpretation of data. V Mautner: revising the manuscript for content, study concept, interpretation of data. SR Plotkin: revising the manuscript for content, study concept, interpretation of data. TY Poussaint: revising the manuscript for content, study concept, interpretation of data. K Robertson: revising the manuscript for content, study concept, interpretation of data. C-S Shih: revising the manuscript for content, study concept, interpretation of data. BC Widemann: revising the manuscript for content, study concept, interpretation of data.

\section{STUDY FUNDING}

This research was supported by the Intramural Research Program of the NIH, National Cancer Institute, Center for Cancer Research.

\section{DISCLOSURE}

E. Dombi reports no disclosures. S. Ardern-Holmes served on a scientific advisory board for Novartis Pharmaceuticals Australia, received funding for a trip from Novartis Pharmaceuticals Australia, and receives research support from the Children's Tumor Foundation of Australia. D. Babovic-Vuksanovic serves as an editorial board member of the Journal of Pediatric Neurology and Acta Medica Academica and received research support from the Children's Tumor Foundation for genetics research. Dr. Babovic-Vuksanovic also served as a scientific reviewer for an NF Clinical Trials Consortium. F. Barker served as a scientific reviewer for the Department of Defense CDMRP panels on neurofibromatosis and tuberous sclerosis. He serves as a section editor for Neurosurgery and has received travel expenses for lectures and educational activities not funded by industry. S. Connor reports no disclosures. D. Evans has served on the scientific advisory boards of the Breast Cancer Campaign and the Genesis Breast Cancer Prevention Appeal. He is on the editorial board of Familial Cancer. He received research support from the Samantha Dickenson Brain Tumor Trust, Cancer Research-UK, the Children's Tumor Foundation, the Association of International Cancer Research, the Medical Research Council, the British Skin Foundation, CLAHRC for Greater Manchester, CMFT RfPB, BDRF, Genome Canada, Genesis Breast Cance Prevention Appeal, and Children with Cancer UK. M. Fisher received reimbursement from the Children's Tumor Foundation to attend their annual Neurofibromatosis Conference, is funded by the Department of Defense (W81XWH-12-1-0155, W81XWH-05-1-0615), Thrasher Research Fund, Children's Tumor Foundation, and Sarcoma Alliance for Research through Collaboration, and received research support from the Pediatric Low Grade Astrocytoma Foundation, Bayer, Children's Discovery Institute, NIH (NR009651-01), and the Department of Defense (W81XWH-08-10051). S. Goutagny reports no disclosures. G. Harris serves on the scientific advisory board of Fovia, Inc. D. Jaramillo serves on the editorial boards of Radiology and Pediatric Radiology. He has received honoraria for speaking engagements from Sheikh Khalifa Medical City, Massachusetts General Hospital, Lucile Packard Children's Hospital, and Boston Children's Hospital He received research support from Genzyme. He also served as a legal case reviewer for Ford and Reminger Co., LPA. M. Karajannis served on a scientific advisory board for Bayer $\mathrm{Ag}$ and on a peer review panel for the Department of Defense/CDMRP Neurofibromatosis Program. He has received honoraria for speaking engagements from the German Cancer Research Center. He has received research support from the NIH as primary investigator on grant R01CA164295 in 2012 and as co-investigator on gran R01CA152975. He also received research support from St. Baldrick's Foundation, Children's Tumor Foundation, Ian's Friends Foundation, Pediatric Low Grade Astrocytoma Foundation, and Making Headway Foundation.
B. Korf serves on the board of directors of the Children's Tumor Foundation, as a senior advisor to the Neurofibromatosis Therapeutic Acceleration Program, and as a member of the board of scientific counselors to the NHGRI. He is on the editorial boards of Genetics in Medicine, Drugs in Context, Journal of Genomes and Exomes, and Current Protocols in Human Genetics. He has received royalties from the publication of Human Genetics and Genomics, Medical Genetics at a Glance, and Current Protocols in Human Genetics from Wiley and from the publication of Principles and Practice of Medical Genetics from Elsevier. He has received honoraria for speaking engagements from Washington University, the American Academy of Pediatrics, Columbus Regional Medical Center, Cambridge Healthtech Institute, the American College of Medical Genetics and Genomics, Case Western University, and KSZ Children's Hospital of Korea. He received research support from Novartis for serving as a principal investigator and he also receives research support from the Children's Tumor Foundation, Department of Defense grants W81XWH-12-1-0155, W81XWH-05-1615, and W81XWH-11-1-0671, and from the NIH/NICHD for grant 2P30HD038985-06A2. V. Mautner reports no disclosures. S. Plotkin has been reimbursed by the American Academy of Neurology and the Children's Tumor Foundation for travel for educational activities. He has received research support from the Department of Defense (W81XWH-091-0182, NF050202, W81XWH-12-1-0155, PI), the Children's Tumor Foundation (PI), and Johns Hopkins Medical Institutes (site PI). T. Poussaint receives research support as the principal investigator of the Neuroimaging Center of the Pediatric Brain Tumor Consortium (NIH-NCI U01CA81456-14). She is associate editor for Pediatric Neuroradiology, as well as Neurographics, She is also a member of the NCI Clinical Imaging Steering Committee. K. Robertson receives research support from the Department of Defense (NF080099 and NF100031). C. Shih received research support from the Department of Defense, award number W81XWH-11-1-0137- DOD, and from Pfizer, grant number LMID L1102860. B. Widemann is a member of the scientific advisory board of the Neurofibromatosis Therapeutic Acceleration Program. She is a member of the editorial board of The Oncologist and an associate editor of Frontiers in Pediatric Oncology. Go to Neurology.org for full disclosures.

Received May 17, 2013. Accepted in final form August 13, 2013.

\section{REFERENCES}

1. Eisenhauer EA, Therasse P, Bogaerts J, et al. New response evaluation criteria in solid tumours: revised RECIST guideline (version 1.1). Eur J Cancer 2009;45:228-247.

2. Miller $A B$, Hoogstraten $B$, Staquet $M$, Winkler A. Reporting results of cancer treatment. Cancer 1981;47: 207-214.

3. Wen PY, Macdonald DR, Reardon DA, et al. Updated response assessment criteria for high-grade gliomas: response assessment in neuro-oncology working group. J Clin Oncol 2010;28:1963-1972.

4. van den Bent MJ, Wefel JS, Schiff D, et al. Response assessment in neuro-oncology (a report of the RANO group): assessment of outcome in trials of diffuse lowgrade gliomas. Lancet Oncol 2011;12:583-593.

5. Korf BR. Plexiform neurofibromas. Am J Med Genet 1999;89:31-37.

6. Kim A, Gillespie A, Dombi E, et al. Characteristics of children enrolled in treatment trials for NF1-related plexiform neurofibromas. Neurology 2009;73:1273-1279.

7. Prada CE, Rangwala FA, Martin LJ, et al. Pediatric plexiform neurofibromas: impact on morbidity and mortality in neurofibromatosis type 1. J Pediatr 2012;160:461-467.

8. Gupta A, Cohen BH, Ruggieri P, Packer RJ, Phillips PC. Phase I study of thalidomide for the treatment of plexiform neurofibroma in neurofibromatosis 1. Neurology 2003;60:130-132.

9. Widemann BC, Salzer WL, Arceci RJ, et al. Phase I trial and pharmacokinetic study of the farnesyltransferase inhibitor tipifarnib in children with refractory solid tumors 
or neurofibromatosis type I and plexiform neurofibromas. J Clin Oncol 2006;24:507-516.

10. Solomon J, Warren K, Dombi E, Patronas N, Widemann B. Automated detection and volume measurement of plexiform neurofibromas in neurofibromatosis 1 using magnetic resonance imaging. Comput Med Imaging Graph 2004;28:257-265.

11. Harris GJ, Plotkin SR, Maccollin M, et al. Three-dimensional volumetrics for tracking vestibular schwannoma growth in neurofibromatosis type II. Neurosurgery 2008;62:13141319; discussion 1319-1320.

12. Weizman L, Hoch L, Ben Bashat D, et al. Interactive segmentation of plexiform neurofibroma tissue: method and preliminary performance evaluation. Med Biol Eng Comput 2012;50:877-884.

13. Babovic-Vuksanovic D, Ballman K, Michels V, et al. Phase II trial of pirfenidone in adults with neurofibromatosis type 1. Neurology 2006;67:1860-1862.

14. Poussaint TY, Jaramillo D, Chang Y, Korf B. Interobserver reproducibility of volumetric MR imaging measurements of plexiform neurofibromas. AJR Am J Roentgenol 2003;180:419-423.

15. Cai W, Kassarjian A, Bredella MA, et al. Tumor burden in patients with neurofibromatosis types 1 and 2 and schwannomatosis: determination on whole-body MR images. Radiology 2009;250:665-673.

16. Jakacki RI, Dombi E, Potter DM, et al. Phase I trial of pegylated interferon-alpha-2b in young patients with plexiform neurofibromas. Neurology 2011;76:265-272.

17. Plotkin SR, Halpin C, McKenna MJ, Loeffler JS, Batchelor TT, Barker FG 2nd. Erlotinib for progressive vestibular schwannoma in neurofibromatosis 2 patients. Otol Neurotol 2010;31:1135-1143.

18. Karajannis MA, Legault G, Hagiwara M, et al. Phase II trial of lapatinib in adult and pediatric patients with neurofibromatosis type 2 and progressive vestibular schwannomas. Neuro Oncol 2012;14:1163-1170.

19. Babovic-Vuksanovic D, Widemann BC, Dombi E, et al. Phase I trial of pirfenidone in children with neurofibromatosis 1 and plexiform neurofibromas. Pediatr Neurol 2007;36:293-300.

20. Widemann B, Dombi E, Gillespie A, et al. Phase II randomized, flexible cross-over, double-blinded, placebocontrolled trial of the farnesyltransferase inhibitor (FTI) tipifarnib (R115777) in pediatric patients (pts) with neurofibromatosis type 1 (NF1) and progressive plexiform neurofibromas (PN). In: Children's Tumor Foundation Conference; 2009; Portland, OR, 2009.

21. Dombi E, Solomon J, Gillespie AJ, et al. NF1 plexiform neurofibroma growth rate by volumetric MRI: relationship to age and body weight. Neurology 2007; 68:643-647.
22. Nguyen R, Dombi E, Widemann BC, et al. Growth dynamics of plexiform neurofibromas: a retrospective cohort study of 201 patients with neurofibromatosis 1 . Orphanet J Rare Dis2012;7:75.

23. Korf B. Natural history of plexiform neurofibromas in NF1. In: Children's Tumor Foundation Conference 2012; 2012; New Orleans.

24. Evans DG, Moran A, King A, Saeed S, Gurusinghe N, Ramsden R. Incidence of vestibular schwannoma and neurofibromatosis 2 in the North West of England over a 10-year period: higher incidence than previously thought. Otol Neurotol 2005;26:93-97.

25. Parry DM, Eldridge R, Kaiser-Kupfer MI, Bouzas EA, Pikus A, Patronas N. Neurofibromatosis 2 (NF2): clinical characteristics of 63 affected individuals and clinical evidence for heterogeneity. Am J Med Genet 1994;52:450-461.

26. Evans DG, Huson SM, Donnai D, et al. A clinical study of type 2 neurofibromatosis. Q J Med 1992;84:603-618.

27. Slattery WH 3rd, Fisher LM, Iqbal Z, Oppenhiemer M. Vestibular schwannoma growth rates in neurofibromatosis type 2 natural history consortium subjects. Otol Neurotol 2004;25:811-817.

28. Peyre M, Goutagny S, Bah A, et al. Conservative management of bilateral vestibular schwannomas in neurofibromatosis type 2 patients: hearing and tumor growth results. Neurosurgery 2013;72:907-913.

29. Dirks MS, Butman JA, Kim HJ, et al. Long-term natural history of neurofibromatosis Type 2-associated intracranial tumors. J Neurosurg 2012;117:109-117.

30. Plotkin SR, Halpin C, Blakeley JO, et al. Suggested response criteria for phase II antitumor drug studies for neurofibromatosis type 2 related vestibular schwannoma. J Neurooncol 2009;93:61-77.

31. Plotkin SR, Singh MA, O'Donnell CC, Harris GJ, McClatchey AI, Halpin C. Audiologic and radiographic response of NF2-related vestibular schwannoma to erlotinib therapy. Nat Clin Pract Oncol 2008;5:487-491.

32. Meany H, Dombi E, Reynolds J, et al. 18-fluorodeoxyglucose-positron emission tomography (FDG-PET) evaluation of nodular lesions in patients with Neurofibromatosis type 1 and plexiform neurofibromas (PN) or malignant peripheral nerve sheath tumors (MPNST). Pediatr Blood Cancer 2013;60:59-64.

33. Widemann B, Babovic-Vuksanovic D, Dombi E, et al. Phase II trial of pirfenidone in children and young adults with neurofibromatosis type 1 (NF1) and progressive plexiform neurofibromas (PN). In: Children's Tumor Foundation Conference; 2009; Portland, OR, 2009.

34. Weiss B, Fisher M, Dombi E, et al. Phase II study of the mTOR inhibitor sirolimus for non-progressive NF1-associated plexiform neurofibormas: a Neurofibromtosis Consortium Study. In: ISPNO; 2010; Vienna, Austria, 2010: ii43. 


\section{Neurology}

Recommendations for imaging tumor response in neurofibromatosis clinical trials Eva Dombi, Simone L. Ardern-Holmes, Dusica Babovic-Vuksanovic, et al. Neurology 2013;81;S33-S40

DOI 10.1212/01.wnl.0000435744.57038.af

This information is current as of November 18, 2013

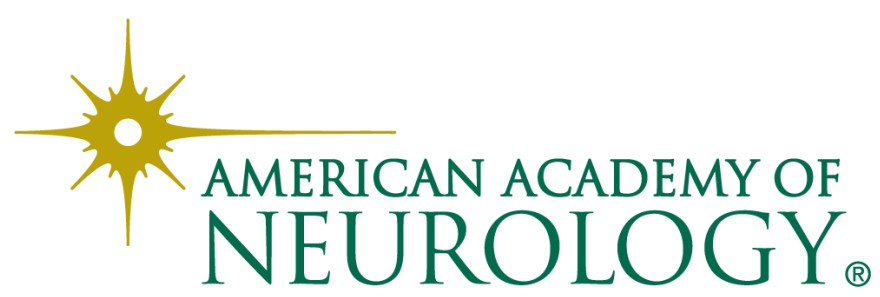




\section{Updated Information \& Services}

\section{Supplementary Material}

\section{References}

Citations

Subspecialty Collections

\section{Permissions \& Licensing}

Reprints including high resolution figures, can be found at:

http://n.neurology.org/content/81/21_supplement_1/S33.full

Supplementary material can be found at:

http://n.neurology.org/content/suppl/2013/11/16/81.21_supplement_1.S 33.DC1

http://n.neurology.org/content/suppl/2013/11/16/81.21_supplement_1.S 33.DC2

This article cites 30 articles, 2 of which you can access for free at: http://n.neurology.org/content/81/21_supplement_1/S33.full\#ref-list-1

This article has been cited by 4 HighWire-hosted articles: http://n.neurology.org/content/81/21_supplement_1/S33.full\#\#otherarti cles

This article, along with others on similar topics, appears in the following collection(s):

\section{All Clinical Neurology}

http://n.neurology.org/cgi/collection/all_clinical_neurology

Clinical trials Methodology/study design

http://n.neurology.org/cgi/collection/clinical_trials_methodology_study design

\section{Nerve tumor}

http://n.neurology.org/cgi/collection/nerve_tumor

Neurofibromatosis

http://n.neurology.org/cgi/collection/neurofibromatosis

Volumetric MRI

http://n.neurology.org/cgi/collection/volumetric_mri

Information about reproducing this article in parts (figures,tables) or in its entirety can be found online at:

http://www.neurology.org/about/about_the_journal\#permissions

Information about ordering reprints can be found online:

http://n.neurology.org/subscribers/advertise

Neurology ${ }^{\circledR}$ is the official journal of the American Academy of Neurology. Published continuously since 1951, it is now a weekly with 48 issues per year. Copyright @ 2013 American Academy of Neurology. All rights reserved. Print ISSN: 0028-3878. Online ISSN: 1526-632X.

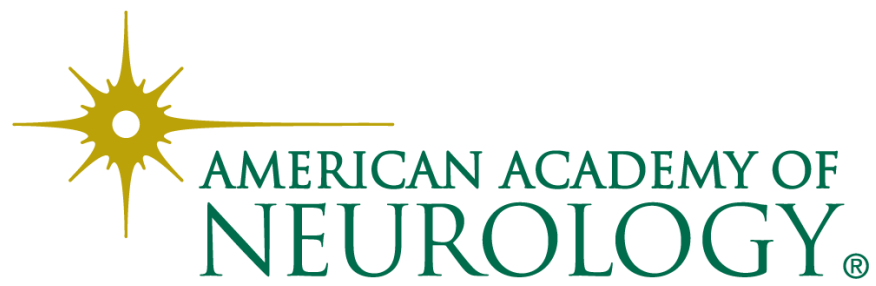

